\title{
Inclusão da reativação da doença de Chagas como uma condição definidora de AIDS para fins de vigilância epidemiológica no Brasil
}

\author{
Inclusion of Chagas' disease reactivation as a condition for AIDS \\ case definition to epidemiological surveillance in Brazil
}

\section{Senhor Editor:}

Reconhece-se que para 0 estabelecimento das ações de vigilância epidemiológica são necessárias estratégias bem definidas para a coleta contínua e sistemática de dados, bem como para a sua adequada análise e interpretação. 0 objetivo principal é a pronta identificação de eventos relacionados ao processo saúde-doença que requeiram ações bem definidas de saúde pública associadas à avaliação de programas ${ }^{1}$.

Adefinição de caso em epidemiologia representa uma dessas estratégias, possibilitando a identificação de indivíduos que apresentam um agravo ou doença de interesse de forma a padronizar critérios para o monitoramento das condições de saúde e para a descrição da ocorrência de uma doença ou agravo sob investigação. 0 objetivo principal é tornar comparáveis os critérios diagnósticos que regulam a entrada de casos no sistema, tanto no nível nacional quanto internacional ${ }^{11}$.

Entretanto, o processo de definição de caso não é estático. Do ponto de vista da vigilância epidemiológica, a definição de caso pode se modificar ao longo do tempo devido à expansão dos conhecimentos clínicos específicos relacionados aos aspectos clínicos e de avaliação complementar, às alterações epidemiológicas e à intenção de ampliar ou reduzir os parâmetros de entrada de casos no sistema, aumentando ou diminuindo sua sensibilidade e especificidade, de acordo com as etapas e as metas estabelecidas por um programa de controle ${ }^{13}$.

Como reflexo desse processo dinâmico, a definição de caso de síndrome da imunodeficiência adquirida (AIDS) vem passando por diferentes modificações nas últimas duas décadas não apenas no Brasil, mas também em outras partes do Mundo. No caso do Brasil, a AIDS tornou-se uma doença de notificação compulsória em 22 de dezembro de 1986, por meio da Portaria no 542 do Ministério da Saúde, juntamente com a sífilis congênita. Desde então, já houve quatro revisões para os casos de AIDS em adultos e três em crianças.
Entrou em vigor em janeiro de 2004 a nova definição de caso de AIDS em adultos e em crianças. Essa nova definição de caso foi o resultado de reuniões do Comitê Assessor de Epidemiologia do Programa Nacional de DST/AIDS realizadas em 2003 e que contaram com a importante participação de representantes da Sociedade Brasileira de Medicina Tropical (SBMT), da Sociedade Brasileira de Doenças Sexualmente Transmissíveis (DST) , da Sociedade Brasileira de Infectologia (SBI), da Sociedade Brasileira de Pediatria (SBP) e da Federação Brasileira das Sociedades de Ginecologia e Obstetrícia ( FEBRASG0).

De uma forma geral, especificamente em relação à definição de caso de AIDS em indivíduos com treze anos de idade ou mais, os principais critérios de definição de caso de AIDS foram revistos, mantendo-se o Critério Rio de Janeiro/Caracas sem qualquer alteração, tendo em vista a sua aplicabilidade e validação anterior, e introduzindo-se adaptações e ajustes na evidência clínica de imunodeficiência estabelecidas no Critério CDC Mbdificado, que passou, dessa maneira, a ser denominado Critério CDC Adaptado. Além disso, os critérios excepcionais foram revistos restando apenas o Critério Excepcional Óbito. Para os menores de treze anos de idade, a simplificação dos critérios resultou em dois critérios: 0 Critério CDC Adaptado e 0 Critério Excepcional Óbito.

Uma das questões discutidas desde a segunda revisão da definição de caso de AIDS em adultos de 1992, era a sinalização de que algumas doenças endêmicas no Brasil como a leishmaniose, a doença de Chagas e a paracoccidioidomicose poderiam ter comportamento oportunista em indivíduos infectados pelo vírus da imunodeficiência humana (HIV) indicando a necessidade de atenção especial dos serviços de saúde, em termos da assistência e da vigilância, visando 0 estabelecimento de evidências que permitissem incluí-las no futuro como indicativas de AIDS4.

1. Departamento de Saúde Comunitária da Universidade Federal do Ceará e Membro do Comitê Assessor de Epidemiologia do Programa Nacional de DST/AIDS do Ministério da Saúde, Brasília, DF, Brasil.

Endereço para correspondência: Dr. Alberto Novaes Ramos Júnior. R. Professor Costa Mendes 1608/50 andar, Rodolfo Teófilo, 60430-140 Fortaleza, CE. Tel: 5585 288-8044, Fax: 5585 288-8050

e-mail: novaes@ iis.com.br

Recebido para publicação em 15/1/2004

Aceito em 25/2/2004 
Aatual revisão da definição de caso de AIDSem adultos cristaliza essas discussões ao avaliar como principal alteração no Critério CDC Adaptado no caso dos adultos a inclusão da reativação da doença de Chagas, expressa clinicamente por meio de miocardite e/ou meningoencefalite, na lista de doenças definidoras. Durante as reuniões do Comitê Assessor de Epidemiologia, avaliou-se a possibilidade de inclusão de outras doenças endêmicas da realidade brasileira, como a leishmaniose visceral, na lista de doenças definidoras. Apesar das possibilidades, tendo em vista a maior complexidade e polimorfismo da co-infecção HIV e Leishmania spp, concluiu-se haver a necessidade de desenvolvimento de estudos mais aprofundados para a validação de critérios visando o estabelecimento da leishmaniose visceral como doença indicativa de AIDS. Além disso, discutiu-se a necessidade de avaliação de formas alternativas de vigilância, frente à elevada prevalência das leishmanioses no Brasil.

Nesse sentido, na presente revisão, optou-se por incluir apenas a reativação da doença de Chagas. Do ponto de vista epidemiológico, a doença de Chagas vem se caracterizando por controle da transmissão vetorial, principalmente a estabelecida pelo Triatoma infestans, ressaltando a importância de outras modalidades anteriormente menos freqüentes, como a transfusional. Entretanto, existe um grande contingente de indivíduos infectados cronicamente pelo Trypanosoma cruzi distribuídos em grande parte do território brasileiro, havendo superposição de áreas em relação à infecção pelo HIV.

Essa inclusão justifica-se tendo em vista os relatos cada vez mais freqüentes na literatura com evidências clínicas e epidemiológicas da reativação dessa condição em pacientes com aids e com outras formas de imunossupressão. Apesar de o processo de reativação da doença de Chagas ser incomum, encontra na infecção pelo HIV em fase avançada um espaço para expressão mais freqüente. Nessa situação, a grande maioria dos casos publicados ${ }^{5710}$ queincluiu a avaliação da contagem de linfócitos T CD4+ tinha esse parâmetro laboratorial inferior a 200 células $/ \mathrm{mm}^{3}$. Acrescenta-se ainda o fato da marcante especificidade da expressão clínica da reativação da doença de Chagas por meio de quadros clínicos de meningoencefalite e miocardite chagásicas agudas ${ }^{2489}$.

Ressalta-se que do ponto de vista da vigilância epidemiológica, não se espera que a inclusão dessa condição represente um fator para aumento significativo da sensibilidade ou da especificidade do critério, apresentando um caráter principal de possibilitar uma maior visibilidade a essa condição específica no sistema de saúde brasileiro. Entretanto, da mesma forma, várias condições presentes na lista original de doenças e agravos dos Centers for Disease Control and Prevention indicativos de AIDS que foram incorporados nas últimas décadas não apenas aos critérios brasileiros, mas também aos de outros países, não apresentaram impacto do ponto de vista epidemiológico para a captação de casos.
Aincorporação da doença de Chagas representa, portanto, 0 amadurecimento das ações de vigilância frente à realidade tipicamente brasileira e 0 estímulo à necessária aproximação entre vigilância e assistência dentro da área das doenças infecciosas e parasitárias. Deve ser encarada ainda como uma importante estratégia tanto para 0 reconhecimento de sua importância bem como para 0 avanço das ações de intervenção e de controle mais específicas da epidemia de infecção pelo HIV/AIDS no Brasil.

\section{Alberto Novaes Ramos Júnior}

\section{REFERÊNCIAS BIBLIOGRÁFICAS}

1. Buehler JH. Surveillance. In: Rothman KJ, Greenland S (eds) Modern Epidemiology. $2^{\text {nd }}$ edition Lippincot-Raven: Philadelphia, p. 435-457, 1998.

2. Ferreira MS. Chagas' Disease and Immunosuppression. Memórias do Instituto Oswaldo Cruz 94 ( supl I) : 325-327, 1999.

3. Laguardia J, Penna ML. Definição de Caso e Vigilância Epidemiológica. Informe Epidemiológico do SUS 8: 63-66, 1999.

4. Lazo JE, Meneses ACO, Rocha A, Frenkel JK, Marquez JO, Chapadeiro E, Lopes ER. Meningoencefalites toxoplásmica e chagásica em pacientes com infecção com vírus das imunodeficiência humana: diagnóstico diferencial anatomopatológico e tomográfico. Revista da Sociedade Brasileira de Medicina Tropical 31:163-171, 1998.

5. Lima JN. Co-Infecção da Doença de Chagas e da Síndrome da Imunodeficiência Adquirida: Freqüência de Casos em Acompanhamento, Perfil Clínico, Laboratorial e Evolução dos Pacientes Atendidos no Hospital de Clínicas da UNICAMP. Tese de Doutorado, Universidade Estadual de Campinas, Campinas, São Paulo, 2001.

6. Ministério da Saúde. Secretaria de Assistência à Saúde. Programa Nacional de Controle de DST/AIDS. Revisão da Definição Nacional de Caso de AIDS em Adultos. Ministério da Saúde, Brasília, Agosto, 1992.

7. Pacheco RS, Ferreira MS, Machado MI, Brito CMM, Pires MQ, Da-Cruz AM, Coutinho SG. Chagas' disease and HIV co-infection: genotypic characterization of the Trypanosoma cruzi strain. Memórias do Instituto Oswaldo Cruz 93: 165-169, 1998.

8. Ramos Jr AN. Reativação da Doença de Chagas: a Associação entre Trypanosoma cruzi e Vírus da Imunodeficiência Humana. Monografia de Conclusão de Residência Médica em Doenças Infecciosas e Parasitárias. Universidade Federal do Rio de Janeiro. Rio de Janeiro, 1999.

9. Rocha A, Ferreira MS, Nishioka SA, Lopes ER. Doença de Chagas: Interação com a Síndrome da Imunodeficiência Adquirida (SIDA). In: Brener Z, Andrade ZA, Barral-Neto M (eds) Trypanosoma cruzi e Doença de Chagas, 2ª edição. Guanabara Koogan, Rio de Janeiro, p. 406-415, 2000.

10. Sartori AMC, Shikanai-Yasuda MA, Neto VA, Lopes MH. Follow-up of 18 patients with human immunodeficiency virus (HIV) infection and chronic Chagas' disease with reactivation of Chagas' disease causing cardiac disease in three patients. Clinical Infectious Diseases 26: 177-179, 1998.

11. Waldman EA. Vigilância em Saúde Pública. São Paulo: Faculdade de Saúde Pública da Universidade Federal de São Paulo; Instituto para 0 Desenvolvimento de Saúde; Núcleo de Assistência Médico-Hospitalar. Série "Saúde e Cidadania", volume 7, 1998.

O documento completo dessa revisão de 2003 (Brasil. Ministério da Saúde. Secretaria de Vigilância em Saúde. Programa Nacional de DST e AIDS. Critérios de definição de casos de AIDS em adultos e crianças. Brasília : Ministério da Saúde, 2003. 50p.) pode ser acessado na homepage do Programa Nacional de DST/AIDS do Ministério da Saúde - www.aids.gov.br 